\title{
A RESPONSABILIDADE SOCIAL EM IES: UMA DIMENSÃO DE ANÁLISE DO SINAES
}

\section{THE SOCIAL RESPONSIBILITY IN IES: A DIMENSION OF ANALYSIS OF THE SINAES}

\author{
Cláudia Medianeira Cruz Rodrigues ${ }^{1}$; José Luis Duarte Ribeiro ${ }^{2}$; Walmir Rufino da Silva ${ }^{3}$ \\ ${ }^{1}$ Universidade Federal da Paraíba - UFPB - João Pessoa/ PB - Brasil cruz2005claudia@hotmail.com \\ ${ }^{2}$ Universidade Federal do Rio Grande do Sul - UFRGS - Porto Alegre/ RS - Brasil ribeiro@producao.ufrgs.br \\ ${ }^{3}$ Universidade Federal da Paraíba - UFPB - João Pessoa/ PB - Brasil walmiruf@uol.com.br
}

\begin{abstract}
Resumo
Este artigo discute o SINAES, Sistema Nacional de Avaliação da Educação Superior, mais especificamente uma das dimensões do Sistema que é a Responsabilidade Social. O SINAES foi instituído pela Lei $n^{o} 10.861$ de 14 de abril de 2004 do Governo Federal e fundamenta-se na necessidade de promover a melhoria da qualidade da educação superior, a orientação da expansão da sua oferta, o aumento permanente da sua eficácia institucional, da sua efetividade acadêmica e social e, especialmente, do aprofundamento dos seus compromissos e responsabilidades sociais. $O$ artigo contribui para a compreensão das premissas e embasamentos do SINAES, assuntos que exigem a atenção de parte das Instituições de Ensino Superior (IES).
\end{abstract}

Palavras-chave: SINAES, Responsabilidade Social, IES

\section{Introdução}

As Instituições de Ensino Superior enfrentam um contexto marcado pela competitividade local, regional e global, pelas exigências governamentais e institucionais, pelos anseios da comunidade acadêmica onde estão inseridas. Diante desse cenário, elas têm buscado uma análise mais aprofundada acerca do desenvolvimento das suas atividades e a conseqüente qualidade delas resultante, tendo em vista a prestação de contas à sociedade, considerando critérios de excelência, eqüidade e relevância social.

Pode-se considerar que a preocupação com a qualidade das IES surge num momento que pode ser caracterizado por um desajuste entre Estado, Universidade e Sociedade. Diversas mudanças em nível social estão demandando novas exigências das universidades, que, por sua vez, não podem responder a tais pronunciamentos sem transformar suas tradicionais estruturas e modos de funcionamento. Neste contexto, surgem diferentes modelos de universidades, assim como 
pressões de diferentes tipos para que se autoavaliem ou sejam avaliadas. Desta forma, a qualidade da educação e a sua avaliação assumem um papel fundamental. Tais mudanças dizem respeito a globalização, expansão e diversificação do sistema de ensino superior, ao surgimento do Estado Avaliador, ao surgimento de novas tecnologias, entre outras.

Empreender uma tarefa de avaliação requer uma noção clara dos elementos que configuram a estrutura da universidade. Isso é necessário em razão de suas particularidades, visto que são organizações com diferenciais próprios, que a convertem em um tipo especial de instituição. Não será possível um conhecimento da totalidade institucional sem o conhecimento prévio das partes que integram essa estrutura e as formas como elas interagem entre si (RODRIGUES, 2003).

O SINAES, Sistema Nacional de Avaliação da Educação Superior, foi instituído pela Lei $\mathrm{n}^{\circ}$ 10.861 de 14 de abril de 2004, do Governo Federal, e têm sido o norteador de todos os instrumentos de avaliação das IES de natureza pública e privada. Atualmente, ele é considerado o principal regulador em termos de verificação da qualidade em suas múltiplas dimensões.

Levando-se em consideração a importância que o SINAES adquiriu nos últimos anos, este artigo discute alguns importantes conceitos que estão relacionados ao tema, como a questão do planejamento institucional e indicadores de desempenho, assim como apresenta de modo específico a dimensão III, que trata da Responsabilidade Social das IES. A metodologia adotada está embasada em referencial teórico, experiência profissional e na legislação vigente, tendo em vista que os estudos e resultados referentes ao sistema ainda estão sendo compilados e apresentados por etapas à comunidade acadêmica.

\section{O SINAES}

O SINAES é um sistema de avaliação global e integrada das atividades acadêmicas, composto por três processos diferenciados: (i) avaliação das IES - AVALIES; (ii) avaliação dos cursos de graduação - ACG e (iii) avaliação do desempenho dos estudantes - ENADE.

Segundo o Art. $2^{\circ}$ da Lei 10.861, o SINAES ao promover a avaliação de instituições, de cursos e de desempenho dos estudantes deverá assegurar:

I. Avaliação institucional, interna e externa, contemplando a análise global e integrada das dimensões, estruturas, relações, compromisso social, atividades, finalidades e responsabilidades sociais das IES e de seus cursos;

II. O caráter público de todos os procedimentos, dados e resultados dos processos avaliativos;

III. O respeito à identidade e à diversidade de instituições e de cursos;

IV. A participação do corpo discente, docente e técnico-administrativo das instituições de educação superior, e da sociedade civil, por meio de suas representações.

No seu Art. $3^{\circ} \mathrm{O}$ SINAES aponta que a avaliação das IES terá por objetivo identificar o seu 
perfil e o significado de sua atuação, por meio de suas atividades, cursos, programas, projetos e setores, considerando as diferentes dimensões institucionais, dentre elas obrigatoriamente as seguintes:

I. A missão e o plano de desenvolvimento institucional.

II. A política para o ensino, a pós-graduação, a extensão e as respectivas formas de operacionalização, incluídos os procedimentos para estímulo à produção acadêmica, as bolsas de pesquisa, de monitoria e demais modalidades.

III. A responsabilidade social da instituição, considerada especialmente no que se refere à sua contribuição em relação à inclusão social, ao desenvolvimento econômico e social, à defesa do meio ambiente, a memória cultural, da produção artística e do patrimônio cultural.

IV. A comunicação com a sociedade.

V. As políticas de pessoal, as carreiras do corpo docente e do corpo técnicoadministrativo, seu aperfeiçoamento, desenvolvimento profissional e suas condições de trabalho.

VI. Organização e gestão da instituição, especialmente o funcionamento e representatividade dos colegiados, sua independência e autonomia na relação com a mantenedora, e a participação dos segmentos da comunidade universitária nos processos decisórios.

VII. Infra-estrutura física, especialmente a de ensino e de pesquisa, biblioteca, recursos de informação e comunicação.

VIII. Planejamento e avaliação, especialmente os processos, resultados e eficácia da autoavaliação institucional.

IX. Políticas de atendimento aos estudantes.

X. Sustentabilidade financeira, tendo em vista o significado social da continuidade dos compromissos na oferta da educação superior. (MEC/INEP, Roteiro de Autoavaliação institucional, 2004)

\section{Referencial Teórico}

A seguir serão apresentados alguns temas que são fundamentais para a compreensão do SINAES, tendo em vista que o Sistema estimula as IES a pensar o processo de avaliação integrado ao planejamento institucional, como pode ser verificado na Dimensão VIII apresentada anteriomente.

A partir da análise do SINAES, verifica-se a preocupação do Governo Federal em apresentar indicadores não somente quantitativos, parâmetro esse utilizado anteriormente pelos processos 
avaliativos, mas também indicadores qualitativos no seu levantamento das dimensões a serem avaliadas.

Muitos desafios são impostos as Instituições neste novo cenário, tendo em vista que mudanças de concepções são requeridas a fim de concluir as etapas as quais as mesmas são solicitadas. Em um primeiro momento, coube as IES realizar o seu processo de auto-avaliação institucional, onde constituiram Comissões Próprias de Avaliações com representações diversificadas: corpo discente, corpo docente, corpo técnico-administrativo e sociedade civil organizada. Os relatórios, a partir do levantamento e análise das dez dimensões, já foram concluídos e entregues ao Governo. No momento, algumas instituições já estão recebendo a visita da comissão de avaliação externa, enquanto outras estão aguardando esta etapa como meio de obter o feedback sobre o que foi desenvolvido.

\subsection{Planejamento em IES}

Tachizawa e Andrade (1999, p.39) questionam: "seria uma instituição de ensino uma organização como as que estamos acostumados a conviver em nosso dia-a-dia?”. Segundo esses autores, assim como qualquer outra organização, estas têm por objetivo satisfazer as necessidades de seus clientes. Devem prestar serviços qualificados de ensino, pesquisa, extensão e, concomitantemente, assegurar um bom ambiente de trabalho para seus funcionários.

No entender de Drucker (1975, p. 177), a universidade é uma instituição de serviço que possui finalidades, valores e objetivos únicos. Para o autor, ela seria caracterizada como uma instituição normativa, que "almeja fazer que seus clientes sejam alguma coisa, façam alguma coisa, comportem-se de determinado modo, saibam certas coisas ou acreditem em certas coisas".

Andrade (2003) enfatiza que as universidades possuem metas a cumprir, necessitam utilizar uma variedade de recursos, formular estratégias e desenvolver sistemas de planejamento para alcançar os resultados desejados, o que exige um aperfeiçoamento constante em seus processos de gestão. Esse mesmo autor aponta que o planejamento nas organizações universitárias não pode ocorrer de forma centralizada, através de um processo desenvolvido de cima para baixo. É necessário considerar que a definição da missão, dos objetivos e das ações necessárias para alcançálos implica a participação de muitas pessoas com interesses divergentes, localizadas em diferentes unidades que constituem 'sistemas autônomos', tais como, faculdades, centros, departamentos e institutos de pesquisa.

Dias (2002) enfatiza que questões como as que tratam do estabelecimento ou revisão de um sistema de avaliação e acreditação, regras para o financiamento público, planos de pós-graduação vinculados às prioridades do desenvolvimento humano do país, política de democratização do acesso que considere o estabelecimento de vínculos mais fortes entre todos os níveis de educação, 
política clara de formação de recursos humanos, política universitária que vise a melhoria da gestão, da capacidade pedagógica dos docentes, do desenvolvimento da cooperação, são elementos que devem ser considerados na discussão e ajudariam aos estabelecimentos de educação superior a definir melhor sua missão.

Conforme o autor, é preciso que sejam examinadas as funções das IES neste contexto de globalização e, ao mesmo tempo, as responsabilidades específicas de cada instituição com relação ao seu entorno geográfico, cultural e social (DIAS, 2002).

Para Tachizawa e Andrade (1999, p. 24), o êxito de uma instituição de ensino no cumprimento de sua missão é a formação de profissionais, com um conjunto de habilidades, competências e conhecimentos, valorizados e reconhecidos pelas organizações que os contratam. No entender desses autores, seja qual for a dimensão em que se considere o cliente, o importante é conhecer aqueles para os quais a instituição de ensino existe, visto que "não há IES que sobreviva se as expectativas de seus clientes não forem ouvidas, interpretadas e atendidas".

Para que isso ocorra, as instituições de ensino devem pesquisar e aferir, junto ao mercado que absorve o aluno formado, os atributos e o perfil do profissional desejado. Do mesmo modo, o aluno formado deve ser consultado para avaliar o resultado que a instituição está obtendo com esse profissional no mercado, considerando que tais resultados são inerentes à missão da instituição. Quanto maior a integração entre a instituição e seus clientes, e quanto maior o sucesso da instituição em unir os interesses dos alunos aos objetivos definidos no seu plano estratégico e projeto pedagógico, melhor serão os resultados referentes ao cumprimento da missão e sobrevivência da instituição.

De acordo com a conferência mundial sobre a Educação Superior no Séc. 21 (documento de trabalho), realizada em Paris no período de 5 a 9 de outubro de 1998, sob o ponto de vista do gerenciamento, uma IES pode ser considerada como um sistema global, composto internamente por subsistemas em interação e envolvendo interações complexas com o mundo exterior, conforme pode ser visualizado na Figura 1.

Uma IES interage, em primeiro lugar, com o meso-ambiente (local e nacional), os quais impõem certas exigências sobre a instituição de ensino (como regulamentos) e provê a ela certos recursos (proporções de seus fundos). Contudo, a IES está inserida em um macro-ambiente que age como um veículo para determinados fenômenos geo-políticos que exercem pressão sobre a mesma. Em contrapartida, a IES influencia esses diferentes ambientes, através de sua contribuição educacional.

Do ponto de vista seqüencial, dois sistemas governam o processo de transformação: (i) aquele denominado de sistema de admissão de alunos, que constituem a matéria-prima das IES, e (ii) o sistema de validação, que especifica as características que esta matéria-prima deve possuir 
quando deixar a instituição.

Visto por essa forma de cruzamento, o sistema global existe e desenvolve-se de acordo com cinco sistemas fundamentais: tarefas, estruturas, recursos, cultura e gestão, sendo que, o sistema de gestão determina se os sete sistemas que compõem o sistema global interagem facilmente. 
Figura 1 - Micro, meso e macro-ambiente que envolvem as IES

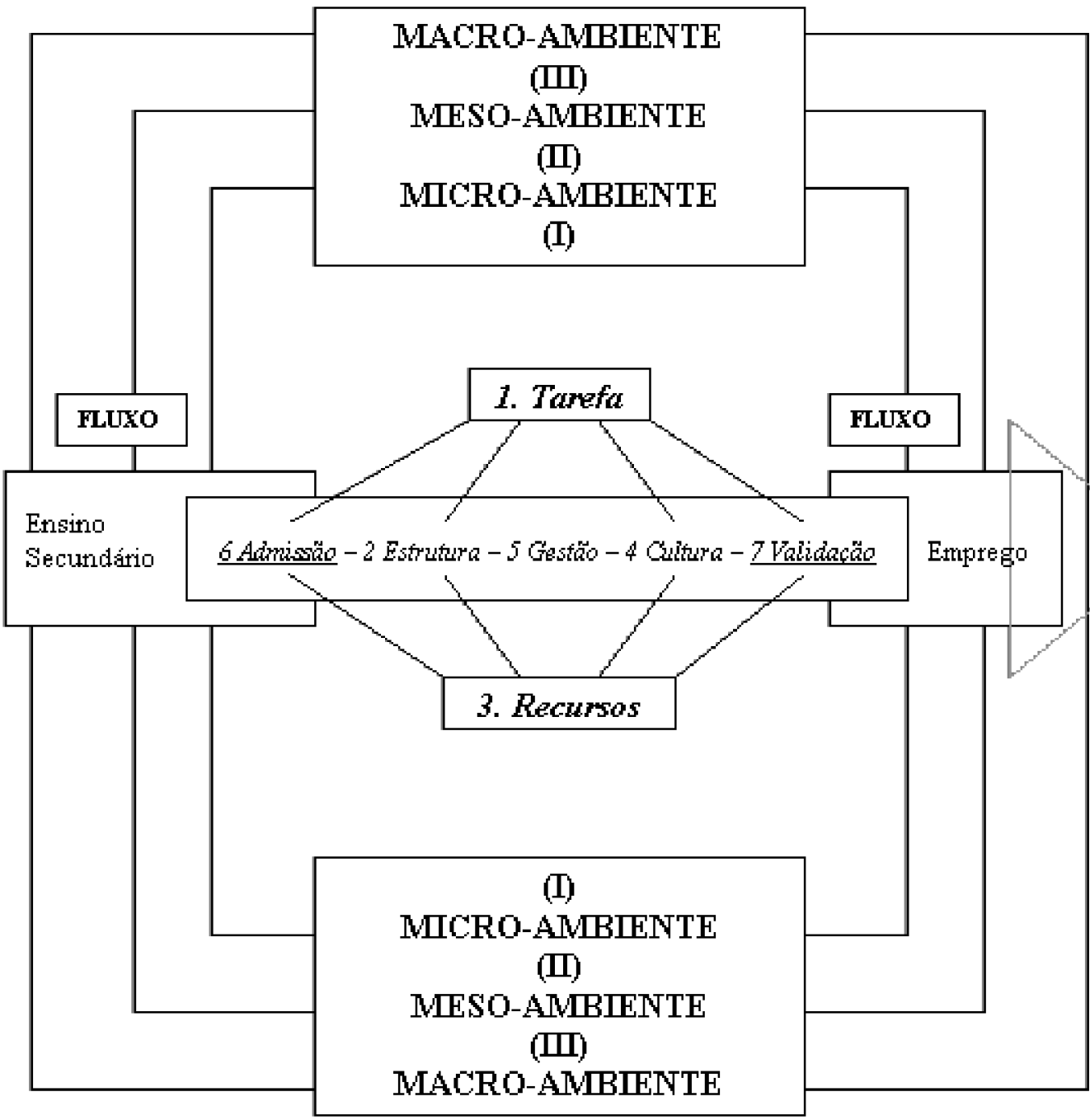

Chave:

- em negrito/italico: sub-sistema da educação superior.

- en sublinhado negrito/italico: sub-sistema na interface de neso e micro

- em letras notmais: sub-sistema meso

- em negrito caixa alta: os diferentes niveis do ambiente ou sub-sistemas.

Fonte: World Conference on Higher Education. In: DIAS (2002, p. 159) 


\subsection{Uso e seleção de indicadores}

Quotidianamente, as pessoas estão sujeitas a avaliação de seu desempenho, tanto em relação ao trabalho, aos estudos, ao comportamento, etc. Ao proceder a avaliação, é preciso que existam indicadores que auxiliem o processo, de modo a fazê-lo da forma mais justa possível, onde, acima de tudo, haja o respaldo em relação aos critérios que serão utilizados.

É preciso que exista uma forma de monitoramento constante que considere tanto aspectos qualitativos quanto quantitativos. Os indicadores, enquanto unidades de medidas, permitem que o monitoramento, o controle e a avaliação possam ser feitos em bases contínuas e mais realistas (KLOECKNER, 2002).

No entender de Cordeiro, Valentina e Possamai (2001, p. 3), o sistema de indicadores deve ser projetado com base numa arquitetura lógica, e não ser apenas uma mera coletânea dos indicadores existentes ou disponíveis, devendo ser levado em consideração no desenvolvimento do sistema de indicadores sua efetiva utilização nas operações diárias, sua abrangência para o acompanhamento do desempenho global da organização e a integração das informações. Desta forma, "os indicadores de desempenho deverão ser por um lado, suficientemente simples e compactos, de modo a permitir uma rápida análise, mas, por outro, permitir um fácil desdobramento em níveis mais detalhados, de modo que possibilite um total acompanhamento de todas as perspectivas".

Na visão de Simons (apud Rocha, 2002), são quatro os critérios que devem ser considerados na escolha dos indicadores: (i) factibilidade técnica de monitorar e medir; (ii) entendimento da causa e efeito; (iii) custo de medição e (iv) nível desejado de inovação.

A factibilidade técnica está vinculada à possibilidade dos gerentes terem condições de monitorar o processo diretamente ou alternadamente; o entendimento da causa e efeito está voltado a cadeia de atividades vinculadas a uma saída, como exemplo o treinamento oferecido ao trabalhador; o custo de medição está vinculado ao resultado que se pode obter com esta medição, ou seja, quanto se pode transformar em resultado efetivo as informações obtidas e por último, o nível desejado de inovação que pode estar vinculado com questões ligadas à qualidade, eficiência ou segurança, sendo o mais importante a ser considerado numa perspectiva gerencial (SIMONS apud ROCHA, 2002).

Hronec (1994) coloca que as medidas de desempenho são sinais vitais da organização, porque quantificam o modo como as atividades em um processo ou output de um processo atingem uma meta específica. Segundo esse autor, as medidas de desempenho não apenas examinam processos e identificam problemas, mas, também, contribuem para prever e evitar problemas. 
O desempenho organizacional pode ser medido sob diferentes óticas: financeira, qualidade, social, tecnológica, entre outras, tendo como propósito identificar se 'as coisas vão bem' dentro da organização e, para isto, há a necessidade da existência de um parâmetro de comparação. Segundo Rocha (2002), para cada necessidade estratégica, podem ser formulados um ou mais indicadores para acompanhar o desempenho da opção estratégica estabelecida, o que pode ser feito através de acompanhamento de diferentes modelos de avaliação de desempenho, ou seja, indicadores contábeis, indicadores de custos, indicadores econômico-financeiros, indicadores da qualidade, indicadores logísticos, balanced scorecards, entre outros.

\subsection{A dimensão III do SINAES - A responsabilidade social}

As dimensões de avaliação do SINAES apresentam indicadores a serem levantados de cunho quantitativo e qualitativo. Cada dimensão apresenta um núcleo de temas básico e comum e um núcleo de temas optativos. Além disso, a documentação e dados a serem observados por cada IES. Tais dimensões podem ser verificadas na íntegra no site: http://www.inep.gov.br.

A seguir apresenta-se os itens a serem avaliados do núcleo básico e comum.

- Transferência de conhecimento e importância social das ações universitárias e impacto das atividades científicas, técnicas e culturais para o desenvolvimento regional e nacional;

- Natureza das relações com os setores público e produtivo, com o mercado de trabalho e com instituições sociais, culturais e educativas de todos os níveis;

- Ações voltadas ao desenvolvimento da democracia, promoção da cidadania, de atenção a setores sociais excluídos, políticas de ações afirmativas, etc.

O núcleo de temas optativos levam em conta ações desenvolvidas pela IES no sentido de inclusão e assistência a setores ou grupos sociais discriminados; critérios adotados para portadores de deficiência especial; atividades em interação com o meio social; políticas institucionais de inclusão de estudantes em situações econômicas desfavorecidas; relações estabelecidas pela instituição com o setor público, com o setor produtivo e com o mercado de trabalho; ações que visem a promoção da cidadania e de atenção a setores sociais; políticas de formação de pesquisadores; entre outros itens muito importantes a serem considerados. (MEC/INEP, Roteiro de Auto-avaliação institucional, 2004).

Verifica-se, através do documento de auto-avaliação, o quanto o Governo está incentivando ações que realmente venham a mostrar através de práticas sociais a real contribuição junto à sociedade, demonstrando a responsabilidade em todas as suas instâncias e/ou particularidades.

Para tanto, afirma Belloni (2000) que é preciso que as instituições educacionais tenham consciência dos objetivos ou funções da avaliação institucional, ou seja, (i) estabelecer mecanismos de controle de qualidade do funcionamento e, principalmente, do produto das instituições, visando 
melhores padrões de eficiência e eficácia; (ii) fornecer informações à própria instituição, ao sistema e à sociedade, com vários objetivos, entre os quais, alocação de recursos humanos e financeiros, formulação de políticas e definição de prioridades e; (iii) institucionalizar um processo de sistemática de reflexão e tomada de decisão com vistas à efetividade social do seu funcionamento, isto é, o cumprimento da missão científica e social da universidade.

Acredita-se que os três objetivos mencionados pela autora remetem a necessidade de integrar a avaliação ao planejamento institucional com responsabilidade social.

\section{Considerações finais}

No Brasil existem atualmente 2.013 IES, sendo 224 públicas (11\%) e 1.789 privadas (89\%), indicando a predominância de instituições privadas no sistema de educação. Tal aspecto pode ser melhor visualizado se for considerado o número de alunos matriculados em ambos os sistemas, visto que de um total de 4.163.733 matrículas, 2.985.405 pertencem ao sistema privado de ensino superior, representando $72 \%$ e somente 1.178 .328 ao sistema público (28\%) (MEC/INEP, Sinopse Estatística da Educação Superior, 2004).

Tais indicadores demonstram a responsabilidade que tais instituições possuem no que tange a oferecer à sociedade produtos e/ou serviços com qualidade, com responsabilidade social, com comprometimento e com respeito às diferenças apresentadas pelos sistemas.

O SINAES, a partir das dimensões a serem avaliadas, proporciona as IES uma oportunidade de mapear seus processos e aprender a verificar o que está sendo efetuado com sucesso e o que precisa ser melhorado ou adaptado a fim de buscar a excelência na prestação dos seus serviços.

Cabe as IES, a partir da análise da legislação em vigor e a partir do direcionamento interno que está embasado no Plano de Desenvolvimento Institucional (PDI), Projeto Pedagógico Institucional (PPI) e Projeto Pedagógico dos Cursos (PPC), alinhar instrumentos de avaliação que consigam levantar, a contento, os indicadores qualitativos e quantitativos que solicita o SINAES na análise do processo de auto-avaliação. No entanto, cabe a cada instituição continuar a história que vem sendo desenvolvida a partir da construção e adaptação de indicadores à sua realidade institucional.

A dimensão enfocada neste artigo é essencial para a vida das instituições, porque promove e estimula a participação, o comprometimento e com isso o retorno social.

A discussão acerca do tema planejamento e indicadores de desempenho se faz necessária na medida em que as instituições necessitam conhecer referenciais que proporcionem condições efetivas para o alinhamento da legislação às práticas adotadas no seu gerenciamento. Mais uma vez salienta-se que as estratégias contruídas pelas IES devem ser preservadas e adaptadas para atender as demandas da sociedade e as exigências da Legislação. 


\begin{abstract}
This paper aims to discuss social responsibility in High Education Institutions or Universities which is an analysis dimension from SINAES (National System for High Education Evaluation) in Brazil. SINAES was established by Law $n^{\circ} 10.861$ from 14 th April 2004, from the Federal Government, viewing to promote the high education quality improvement, to incentive the vacancy offer expansion, to improve permanently the institutional efficacy, social and academic efficiency and, particularly, to give emphasis to the universities social responsibilities and engagements. This paper focuses the elucidation of SINAES premises and bases since these are themes of extreme importance to High Education Institutions administrators.
\end{abstract}

Key-words: SINAES, Social Responsability, High Education Institutions

\title{
Referências
}

ANDRADE, Arnaldo Rosa de. Gestão estratégica de universidades: análise comparativa de planejamento e gestão. In: ENCONTRO ANUAL DA ANPAD - ENANPAD, 27., 2003, Atibaia/SP. Anais... Atibaia, 2003.

BELLONI, Isaura. A função social da avaliação institucional. In: DIAS SOBRINHO, José; RISTOFF, Dilvo I. Universidade desconstruída: avaliação institucional e resistência. Florianópolis: Insular, 2000, p. 37-58.

CORDEIRO, Nadir Radoll; VALENTINA, Luiz V. O. Dalla; POSSAMAI, Osmar. A utilização do Balanced Scorecard na otimização de processos. In: ENCONTRO NACIONAL DE ENGENHARIA DE PRODUÇÃO, 21 E VI INTERNATIONAL CONFERENCE ON INDUSTRIAL ENGENNERING AND OPERATIONS MANAGEMENT, 6. 2001, Salvador/BA. Anais...Salvador, 2001.

DIAS, Marco Antonio Rodrigues. Utopia y comercialización en la educación superior del siglo XXI. In: DIAS, Marco Antonio Rodrigues (Coord.). Perspectivas de la educación superior en el siglo XXI: lecciones de la conferencia mundial sobre educación superior. Madrid: CRUE, p. 11-44, 2002.

DRUCKER, Peter F. Administração, tarefas, responsabilidades, práticas. São Paulo: Pioneira, 1975.

HRONEC, Steven M. Sinais vitais: usando medidas de desempenho da qualidade, tempo e custos para traçar a rota para o futuro de sua empresa. São Paulo: Makron Books, 1994.

KLOECKNER, Mônica Caravantes. Avaliação da readministração em uma IES: um estudo de caso. Porto Alegre, 2002. Dissertação (Mestrado em Engenharia de Produção). Programa de Pós-Graduação em Engenharia de Produção, UFRGS, 2002.

MEC/INEP. SINAES. Roteiro de Auto-avaliação institucional 2004. Brasília/ DF, 2004.

MEC/ INEP. Sinopse estatística da educação superior Censo 2004. Sistema de avaliação da educação superior. Brasília/ DF, 2004.

ROCHA, Augusto Cesar Barreto. Configuração de um sistema de avaliação de desempenho alicerçado no Balanced Scorecard para uma indústria de confecções de porte médio. Florianópolis, 2002. Dissertação. (Mestrado em Engenharia de Produção). Programa de Pós-Graduação em Engenharia de Produção, UFSC, 2002.

RODRIGUES, Cláudia Medianeira Cruz. Proposta de avaliação integrada ao planejamento anual: um modelo para as UCGs. Porto Alegre, 2003. Tese. (Doutorado em Engenharia de Produção). Programa de Pós-Graduação em Engenharia de Produção, UFRGS, 2003.

TACHIZAWA, Takeshy; ANDRADE, Rui Otávio Bernardes de. Gestão de instituições de ensino. Rio de janeiro: Fundação Getúlio Vargas, 1999. 


\section{Dados dos autores:}

Cláudia Medianeira Cruz Rodrigues

UFPB

Ciências Administrativas

Prof. Adjunto

Rua Borges de Medeiros, 526 - Vila Vitória - Santa Maria/ RS, Cep. 97040-000.

Telefones: (055) 3220-9296

e-mail: cruz2005claudia@hotmail.com

José Luis Duarte Ribeiro

UFRGS - DEPROT

Prof. Adjunto

Av. Luiz Manoel Gonzaga 915, casa 2, Porto Alegre/ RS, Cep. 90.470-280.

Telefones: (51) 3316-4005

e-mail: ribeiro@producao.ufrgs.br

Walmir Rufino da Silva

UFPB

Administração

Coordenador do PPGA

Rua Iolanda Henrique Cavalcanti, nº 20/601, Bessa, João Pessoa/ PB, Cep. 58037-120.

Telefones: (83) 3216-7492

e-mail: walmiruf@uol.com.br

Recebido para publicação em: 25/10/2006

Aceito para publicação em: 01/12/2006 